\title{
Pneumonia lipoide exógena em peixe-boi marinho (Trichechus manatus): relato de caso
}

\author{
[Exogenous lipoid pneumonia in manatee (Trichechus manatus): case report]
}

\section{"Relato de Caso/Case Report"}

\author{
Fernanda Loffler Niemeyer Attademo ${ }^{1,2^{*}}$, Augusto Carlos Boaviagem Freire $^{1}$, Glaucia Pereira \\ Sousa $^{2}$, Ieverton Cleiton Correa Silva ${ }^{3}$, Fabia de Oliveira Luna ${ }^{2}$, Fabiano Sellos Costa ${ }^{3}$
}

\author{
${ }^{1}$ Centro de Estudos e Monitoramento Ambiental, Areia Branca-RN, Brasil. \\ ${ }^{2}$ Centro Nacional de Pesquisa e Conservação de Mamíferos Aquáticos, Instituto Chico Mendes de Conservação da \\ Biodiversidade, Santos-SP, Brasil. \\ ${ }^{3}$ Departamento de Medicina Veterinária, Universidade Federal Rural de Pernambuco, Recife-PE, Brasil. \\ *Autora para correspondência/Corresponding author: E-mail: niemeyerattademo@yahoo.com.br
}

\begin{abstract}
Resumo
O peixe-boi-marinho (Trichechus manatus) é um dos mamíferos aquáticos mais ameaçados de extinção do Brasil. A reabilitação e soltura dos filhotes resgatados no litoral do nordeste tem sido uma das principais medidas de conservação para a espécie. Foi realizado o relato de caso de um filhote de peixe-boi resgatado pelo Instituto Chico Mendes de Conservação da Biodiversidade Centro Nacional de Pesquisa e Conservação de Mamíferos Aquáticos, que após alimentação com mistura láctea hiperlipídica contendo óleo mineral, passou a apresentar alterações de flutuabilidade e edemaciação da região ventral. Foram realizados exames hematológicos, comportamentais e de imagem no animal. A tomografia computadorizada indicou uma pneumonia lipoide exógena. Para a descrição do caso, foram utilizados os prontuários clínicos da instituição. O protocolo terapêutico preconizado foi realizado em conjunto com o acompanhamento clínico e, sempre que necessário readequado conforme a evolução do quadro. Durante o tratamento, foram fornecido antibioticoterapia, antiinflamatório, polivitamínicos, probióticos, além da alimentação láctea. Após o tratamento terapêutico, o quadro apresentou involução dos achados clínicos e o animal voltou as condições normais, voltando a fazer parte do plantel de soltura de peixes-bois da instituição. Após este caso clínico, foi suspensa na instituição a adição de óleo de origem mineral na alimentação dos animais, pois considerou-se que este fator pode ter sido um agravo ao caso. Este relato documenta as alterações clínicas, diagnóstico e tratamento adotado para a doença em peixe-boi-marinho.
\end{abstract}

Palavras-chave: reabilitação; óleo mineral; tomografia computadorizada.

\section{Abstract}

Antillean manatees (Trichechus manatus) is one of the most endangered aquatic mammals in Brazil. The rehabilitation and release of the calves rescued on the northeastern coast has been one of the main conservation measures for the species. This manuscript describes the case of a manatee calf rescued by the Chico Mendes Institute for Biodiversity Conservation National Center for Research and Conservation of Aquatic Mammals, which, after being fed with a hyperlipidic milk mixture containing mineral oil, started to present changes in buoyancy and swelling of the ventral region. Hematological, behavioral, and imaging tests were performed on the animal. CAT scan images showed exogenous lipoid pneumonia. For the description of the case, the institution's clinical records were used. The recommended therapeutic protocol was carried out in conjunction with clinical follow-up and, whenever necessary, readjusted according to the evolution of the condition. During treatment, antibiotic therapy, anti-inflammatory drugs, multivitamins, probiotics, in addition to dairy food were provided. After therapeutic treatment, the clinical findings have regressed along with the regular health conditions, and the animal returning to be part of the institution's manatee release squad. After this clinical case, the addition of mineral oil in the animals' diet was suspended at the institution, as it was considered that this factor may have been an aggravation to the case. This report documents the clinical changes, diagnosis and treatment adopted for the disease in an Antillean manatee.

Keywords: rehabilitation; mineral oil; tomography. 


\section{Introdução}

O peixe-boi marinho (Trichechus manatus), pertencente à ordem Sirenia, é um dos mamíferos aquáticos mais ameaçados de extinção do Brasil, estando listado como em ameaça (EN), quanto a sua conservação (Luna et al., 2018). Para minimizar o status de vulnerabilidade da espécie no país, entre as ações estratégicas de proteção, a reabilitação dos animais resgatados no litoral do nordeste (Balensiefer et al., 2017) e posterior soltura em ambiente natural é uma medida eficiente adotada (Luna e Passavante, 2010; Normande et al., 2016).

O longo tempo necessário para cumprir o processo de reabilitação, podendo variar entre três a cinco anos, faz-se necessário o estudo continuado acerca da saúde dos peixes-boi em cativeiro (Vergara-Parente et al., 2003). É de conhecimento que uma vasta diversidade de patógenos como bactérias, vírus e parasitas podem interferir na saúde desses animais (Attademo et al., 2020a).

Doenças do trato respiratório, sejam elas ocasionadas por microrganismos ou causas externas, têm sido verificadas em peixes-bois no Brasil (Vergara-Parente et al., 2003, Attademo et al., 2020a; Attademo et al., 2020b). Em outras espécies de mamíferos aquáticos, como os golfinhos da espécie Pontoporia blainvillei foram verificados em achados de necropsia, pneumonia crônica associada a depósitos de colesterol (CPC) (Ruoppolo et al., 2010). A pneumonia lipoide, vem sendo descrita com maior frequência em seres humanos (Albuquerque-Filho, 2006; Caswell e Williams, 2007). Na medicina veterinária já foi descrita em caninos, podendo estar associada à neoplasias (Pérez-Accino et al., 2021). Entretanto, habitualmente são resultantes da inalação acidental de óleo ou derivado por refluxo gástrico, o que pode acarretar um processo inflamatório intersticial crônico proliferativo do parênquima pulmonar, levando à pneumonia ou mesmo óbito (Albuquerque-Filho, 2006). Nestes casos, a ferramenta de diagnóstico tem sido o histórico clínico, exame de imagem ou análise pós-morte.

O presente estudo teve como objetivo relatar os aspectos clínicos, exames de imagens e tratamentos terapêuticos do primeiro registro de pneumonia lipoide exógena diagnosticada em peixe-boi-marinho, decorrente da ingestão de óleo mineral.

\section{Descrição do Caso}

No dia 10 de outubro de 2014, um neonato de peixe-boi marinho (Trichechus manatus), fêmea, encalhou no município de Maceió, estado de Alagoas, Brasil (9`33'05,69'S; $\left.35^{\circ} 37^{\prime} 39,54^{\prime \prime} \mathrm{O}\right)$. O animal, com $22 \mathrm{~kg}$ de peso corpóreo e $110 \mathrm{~cm}$ de comprimento total, apresentava vestígios do cordão umbilical, desidratação moderada, mucosas normocoradas e ausência de lesões por interação antrópica. Após o resgate, o animal foi transportado para o Centro de Reabilitação do Instituto Chico Mendes de Biodiversidade / Centro Nacional de Pesquisa e Conservação de Mamíferos Aquáticos (ICMBio/CMA), localizado na Ilha de Itamaracá, estado de Pernambuco $\quad\left(7^{\circ} 48^{\prime} 33.40^{\prime \prime} \mathrm{S}\right.$, $\left.34^{\circ} 50^{\prime} 16.36^{\prime \prime} \mathrm{O}\right)$, Brasil. Neste local, recebeu o nome de Paty, em homenagem a praia de Pratagy, local do encalhe.

Após avaliação clínica e estabilização foi fornecido ao neonato alimentação via mamadeira, contendo $200 \mathrm{~mL}$ de água mineral, 24 gramas de proteína isolada de soja (Soymilke ${ }^{\circledR}$ - Olvebra) e 20 gotas de extrato de própolis não alcoólico, acrescido de suplementação vitamínica, mineral e de aminoácidos essenciais: $8 \mathrm{~mL}$ de Glicopan ${ }^{\circledR}$ Vetnil e $2 \mathrm{~mL}$ de Hemolitan $®$ - Vetnil. A mistura láctea foi ofertada cinco vezes ao dia, em intervalos de 2 horas entre as mamadas. $\mathrm{O}$ animal foi mantido em recinto redondo de diâmetro de 3 metros e $6,35 \mathrm{~m}^{3}$ com água salgada de, sendo acoplado uma fonte de água corrente (ad libidum) por meio de mangueira.

Foram realizados exames sanguíneos de hemograma e bioquímicos no terceiro dia após o encalhe, quando se verificou um aumento de hematócritos e bastonetes, além de trombocitopenia e. Estas alterações têm sido compatíveis com os primeiros dias de encalhe de outros filhotes neonatos de peixes-bois atendidos pela instituição, não corroborando com achados patológicos.

No $12^{\circ}$ dia após o encalhe do peixe-boi foi evidenciado um quadro de constipação e cólica. A fim de reverter os sintomas, foram acrescidos em cada uma das cinco mamadeiras 10 $\mathrm{mL}$ de óleo de canola e 50 gotas de simeticona $[75 \mathrm{mg} / \mathrm{mL}]$. Após três dias de tratamento, e persistência dos sinais clínicos, foi acrescido $5 \mathrm{~mL}$ de óleo mineral em cada mamadeira. No terceiro dia após reajuste do protocolo terapêutico instituído, o animal passou a defecar de forma espontânea, porém fezes de consistência 
endurecida e ressecada, mantendo-se as características por mais 10 dias. Diante da hipersensibilidade na região abdominal persistentes, foi acrescido 25 gotas de dipirona [500 mg/mL] VO - SID.

Além do analgésico, foi reformulada a formulação láctea para: $200 \mathrm{~mL}$ de água de coco, $100 \mathrm{~mL}$ de água mineral, 40 gramas de leite de soja Soymilke® - Olvebra, com manutenção da suplementação vitamínica e acrescida 50 gotas de simeticona $[75 \mathrm{mg} / \mathrm{mL}], 10 \mathrm{~mL}$ de óleo mineral, $5 \mathrm{~mL}$ de óleo de canola e $1 \mathrm{~mL}$ de óleo de coco, durante 5 vezes ao dia. Após 10 dias do novo manejo alimentar, 35 dias após encalhe, o peixeboi passou a apresentar estabilização clínica e normalização das necessidades fisiológicas. Com isso, a oferta de óleo mineral e de coco foram suspensas, mantendo apenas o óleo de canola, como única fonte de suplementação lipídica, essencial na dieta alimentar da espécie, em processo de reabilitação.

No dia 27 de novembro de 2014 foram realizados novos exames hematológicos, sendo verificado uma trombocitopenia, eosinofilia, basofilia e bastonetes aumentados, entretanto os hematócritos haviam se estabilizado. Nesta ocasião, foi observada uma área edemaciada na região cervical ventral de consistência levemente flácida (Figura 1), além de sinais de apatia e inapetência.

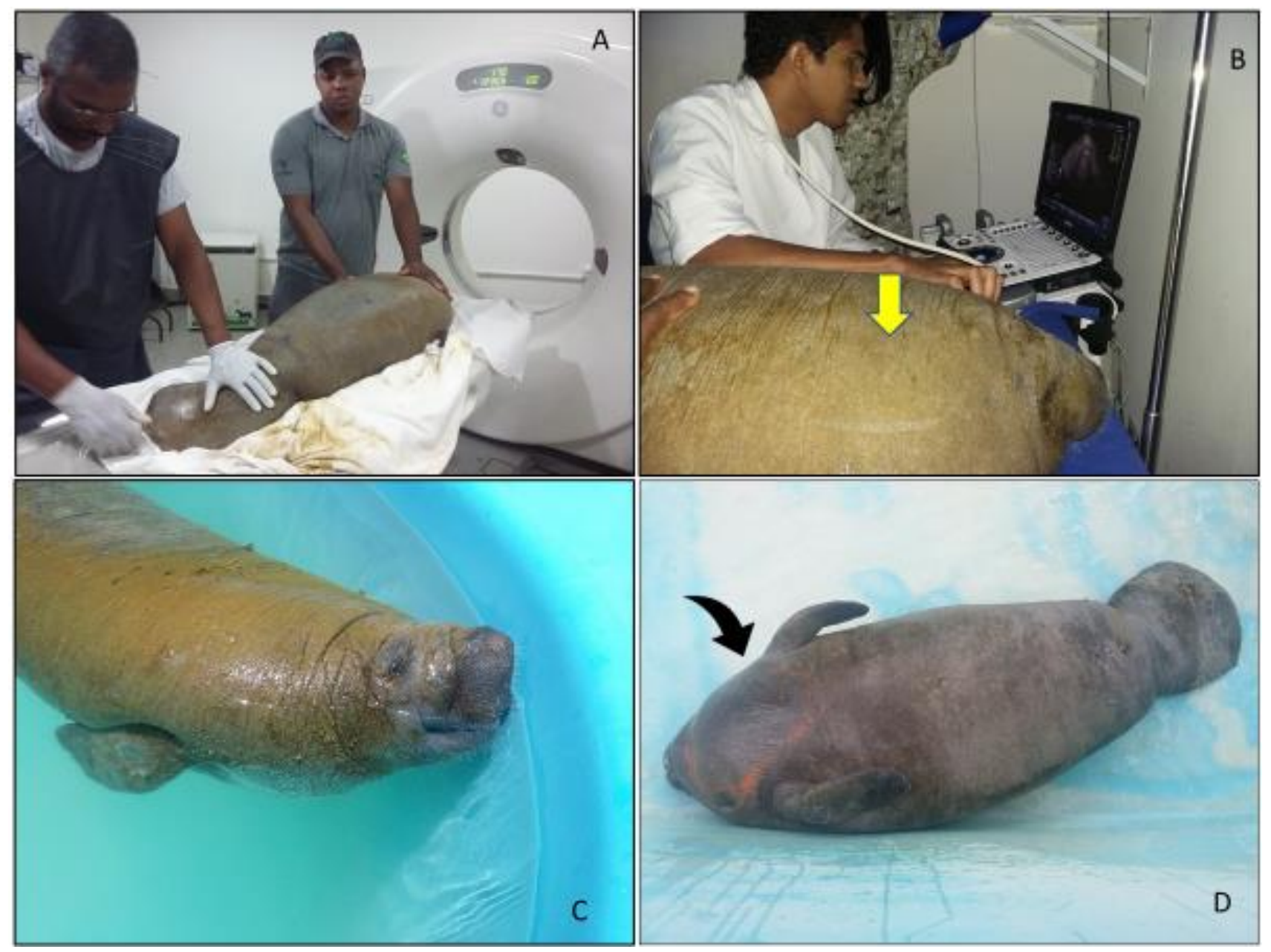

Figura 1. Realização de exames e achados anatômicos de pneumonia lipoide exógena em peixe-boi marinho (Trichechus manatus). (A) Animal posicionado para a realização de exame tomográfico. (B) Animal posicionado para a realização de exame de ultrassonografia e demonstrando região edemaciada e abaulada na lateral direita (seta). (C) Peixe-boi com leve adernação mantendo o lado direito para fora da água. (D) Região edemaciada e abaulada ventralmente.

Diante aos achados físicos e laboratoriais, foi realizado exame de tomografia computadorizada, a fim de melhor compreender e elucidar as possíveis causas e gravidade das alterações. Para obtenção das imagens foi utilizado o tomógrafo Hi-Speed FXI - General Eletrics, 06828-0001, Fairfield, Connecticut single slice, sendo devidamente calibrado antes da 
realização do exame. As imagens foram obtidas por aquisição helicoidal em cortes transversais de $3 \mathrm{~mm}$ de espessura, intervalo entre cortes de 0,8 $\mathrm{mm}$, no sentido craniocaudal, com técnica de exame de $120 \mathrm{Kv}$ e $130 \mathrm{~mA}$. Utilizou-se algoritmo de reconstrução para parênquima pulmonar e tecido ósseo. O peixe-boi foi contido manualmente, não havendo a necessidade de contenção química em nenhum momento durante o curso do exame. As imagens foram avaliadas usando o software visualizador DICOM dedicado (Horus versão 3.3.5) em uma estação de trabalho (Apple Macbook Air, Apple). Para avaliação das imagens foram utilizadas reconstruções multiplanares e janelas de avaliação variáveis de acordo com as preferências do observador.
Diante dos materiais gerados foi possível observar aumento da atenuação radiográfica em porções craniais dos campos pulmonares, com presença de infiltrado intersticial bilateral e área de infiltrado alveolar em porção cranial do lobo pulmonar esquerdo, sendo que nesta região a radiodensidade apresentou valores médios de $109 \mathrm{HU}$. Espessamento de septo interlobular na periferia da lesão também foi acurado.

Associando-se o histórico do paciente com achados clínicos e tomográficos estabeleceuse o diagnóstico presuntivo de pneumonia lipoide, supostamente ocasionada por aspiração da mistura láctea hiperlipídica, utilizada no tratamento dos sinais clínicos de constipação na fase inicial do processo de reabilitação (Figura 2).

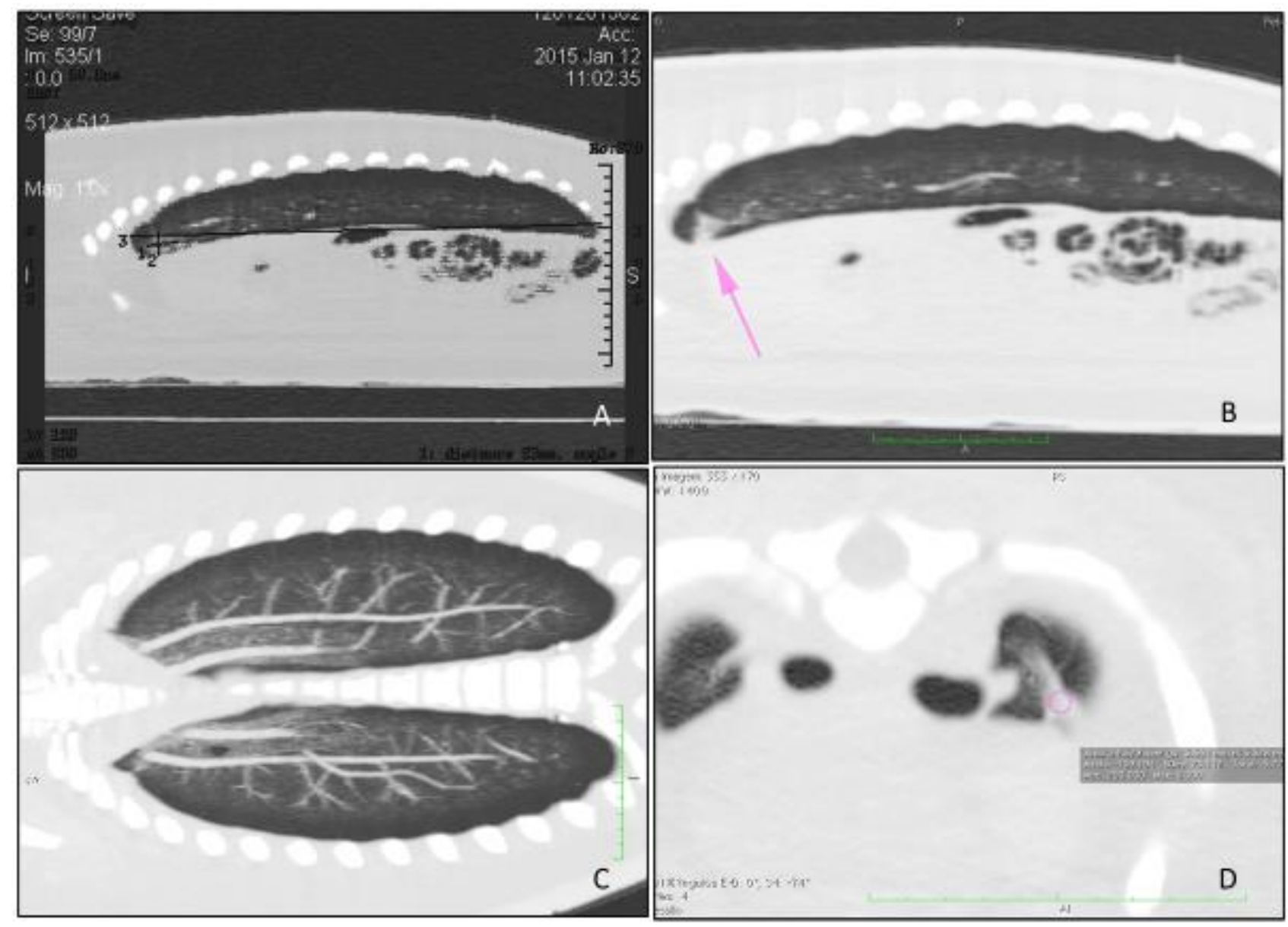

Figura 2. Achados de imagem de pneumonia lipoide exógena em peixe-boi marinho (Trichechus manatus). (A e B) Imagem tomográfica em reconstrução multiplanar sagital, evidenciando lesão atenuante em porção cranial de parênquima pulmonar (seta). (C) imagem de reconstrução multiplanar coronal, evidenciando parênquima pulmonar e vasos pulmonares, sendo possível visualizar opacificação intersticial em lobos craniais. (D) Análise densiométrica pulmonar demonstrando imagem com radio densidade compatível com conteúdo lipídico (círculo).

O tratamento preconizado para a pneumonia lipoide foi à base de benzilpenicilina + procaína + dihidroestreptomicina, associado à piroxicam (Pencivet Plus PPU® - Saúde Animal), na dose de $1 \mathrm{~mL} / 30 \mathrm{~kg}$ de peso vivo, IM - SID, durante 5 dias; furosemida $(2 \mathrm{mg} / \mathrm{kg}) \mathrm{VO}$ - SID, por 12 dias, e probiótico Biobac ${ }^{\circledR}$ - Ouro Fino, 5 gramas BID - VO, por 12 dias, para equilíbrio e 
manutenção da microbiota gastrointestinal. A alimentação passou a ser de $500 \mathrm{~mL}$ de água de coco, 80 gramas de Soymilke® - Olvebra, $2 \mathrm{~mL}$ de óleo de canola, $10 \mathrm{~mL}$ de Glicopan ${ }^{\circledR}$ - Vetnil, $3 \mathrm{~mL}$ de Hemolitan ${ }^{\circledR}$ - Vetnil, 50 gotas de extrato de própolis sem álcool e $5 \mathrm{~mL}$ de mel, cinco vezes ao dia; 5 gramas de $E$-S-E Super ${ }^{\circledR}$ - Vetnil (VO BID) e 5 gramas de Organew ${ }^{\circledR}$ - Vetnil (VO SID).

Após 45 dias de início de todo tratamento, observou-se a remissão dos achados tomográficos identificados no primeiro exame, com o parênquima pulmonar assumindo grau de atenuação radiográfica dentro dos padrões de normalidade para a espécie, além de qualquer sintomatologia clínica. Atualmente o animal encontra-se dentro do plantel de animais aptos para soltura, em recinto de aclimatação, em perfeito estado de saúde, aguardando o término da pandemia do COVID-19 para ser solto à natureza.

\section{Discussão}

Os peixes-bois possuem grande dependência de cuidados parentais e, quando junto às mães, permanecem cerca de dois anos em amamentação contínua (Luna e Passavante et al., 2010). No Brasil, o encalhe de peixes-bois vivos são predominantemente filhotes neonatos, com até uma semana de vida, exigindo cuidados humanos para garantir sua sobrevivência (Luna e Passavante et al., 2010; Balensiefer et al., 2017). Desta forma, quando ocorre um encalhe, os filhotes são resgatados e levados para centro de reabilitações, como por exemplo o ICMBio/CMA, localizado na Ilha de Itamaracá (Luna e Passavante et al., 2010). Ao chegarem, os filhotes recebem um aleitamento artificial formulado de acordo com as necessidades e condição clínica de cada espécime (Gage e Walsh, 2018; Freire et al., 2021). Neste sentido, após o resgate, Paty foi transferida para o ICMBio/CMA, contribuindo para as ações previstas no Plano de Ação Nacional para a Conservação (PAN) do Peixe-boi-marinho (BRASIL, 2018).

A composição nutricional do leite da fêmea de peixe-boi não está bem elucidada, sendo utilizado principalmente bases referenciais de peixes-bois da Flórida Trichechus latirostris (Bachman e Irvine, 1979; Rosen e Worthy, 2018; Gage e Walsh, 2018), que destacam a característica do alimento possuir um alto conteúdo lipídico, especialmente nos primeiros meses de amamentação (Rosen e Worthy, 2018).
O ICMBio/CMA, para suprir as necessidades nutricionais dos filhotes em reabilitação, vêm fornecendo uma dieta à base de proteína de soja, acrescida de fonte lipídica (óleo), polivitamínicos e aminoácidos essenciais, solução a qual vem permitindo que os filhotes tenham uma curva de crescimento e ganho de peso, semelhante aos dos animais que nasceram em cativeiro, sob cuidados maternos e submetidos a amamentação natural (Borges et al., 2012).

Doenças de origem nutricionais e alterações pulmonares vêm sendo parte das principais causas de agravos na saúde em peixesbois (Buergelt et al., 1984; Attademo et al., 2020a). A pneumonia em peixes-bois, assim como na maioria dos mamíferos aquáticos, pode trazer graves problemas à saúde dos animais e inclusive levar ao óbito (Attademo et al., 2020b). Em golfinhos-nariz-de-garrafa (Tursiops truncatus), no Japão, foi relatado óbito de três filhotes por pneumonia aspirativa (Makara et al, 2007). Na ocasião, os autores verificaram que os animais desenvolveram a patologia pelo histórico de fraqueza desde o nascimento, contribuindo assim com a broncoaspiração do leite. No presente estudo, o filhote realizou broncoaspiração do sucedâneo hiperlipídico, levando a um quadro de pneumonia lipoide. Se não houvesse sido preconizado um protocolo terapêutico adequado, possivelmente este animal poderia ter evoluído ao óbito, como ocorrido no caso de piotorax (Attademo et al., 2020).

Por se tratar de animal aquático, a identificação de alteração clínica em peixes-bois pode ser de grande complexidade, sendo necessário o acompanhamento diário do comportamento, associado à exames laboratoriais (hemograma, bioquímico, análise bacteriológica e outros) e quando possível, exames complementares e de imagem. A identificação da evolução do caso relatado, somente foi possível pois desde antes dos sintomas, durante o episódio e após a estabilização do quadro, o animal foi avaliado.

O acompanhamento do quadro clínico e laboratorial também permitiu adequar a dieta láctea artificial da fêmea às necessidades clínicas, não tendo sido desenvolvido alterações hematológicas significativas, sendo possível o tratamento efetivo e seguro. Com isso, permitiu que após a remissão dos achados tomográficos, o animal voltasse a apresentar pleno 
desenvolvimento físico, clínico e de comportamento.

A tomografia computadorizada como ferramenta de diagnóstico na medicina veterinária vem crescendo nos últimos anos, como um método eficaz (Lehmhuhl e Vulcano, 2014). Esta técnica permite a avaliação seccional da região a ser avaliada, apresentando as vantagens de não ser invasiva e não apresentar sobreposição de imagens (Tidwel, 2007). Contudo, a tomografia computadorizada para peixes-bois ainda apresenta restrições, tanto devido à condição fisiológica do animal, quando houver necessidade de instituir um plano anestésico, ou por questões anatômicas, já que estes animais detêm de grande peso e espessa camada de gordura. Neste estudo, a realização do exame foi possível por se tratar de um filhote, de pequeno porte, e com camada de gordura pouco espessa.

Apenas um estudo clínico utilizando tomografia computadorizada em peixe-boi foi descrito, possibilitando um diagnóstico eficaz, de pneumotorax e pneumoperitônio (Gerlach et al., 2013). Porém, nenhum outro relato de pneumopatia foi descrito ao conhecimento dos autores. O diagnostico sugerido de pneumonia lipídica foi sugerido pelas lesões no parênquima e pela observação de imagem com atenuação radiográfica compatível com marcadores e densidade de conteúdo lipídico, tendo em vista que a radiodensidade do tecido adiposo oscilar entre -150 e -50 unidades Hounsfield (Saunders e Schwarz, 2011).

\section{Conclusão}

O protocolo preconizado se mostrou eficiente para o tratamento da pneumonia lipoide exógena em peixe-boi marinho. $\mathrm{O}$ uso da ferramenta de diagnóstico de imagens para avaliação clínica de filhotes de peixe-boi pode ser eficiente e fornecer respostas rápidas sobre a origem do problema, permitindo um rápido tratamento. Após este caso, foi suspensa a adição de óleo de origem mineral na alimentação dos animais, pois considerou-se que este fator pode ter sido um agravo ao caso. Desde então, como fonte lipídica na dieta, vem sendo utilizado apenas óleo de origem vegetal, tal como óelo de canola ou óleo de coco.

\section{Conflitos de interesse}

Os autores declaram não existir conflito de interesses. O presente estudo foi realizado por meio da licença SISBIO Nº 20685.

\section{Agradecimentos}

Ao Instituto Chico Mendes de Conservação da Biodiversidade / Centro Nacional de pesquisa e Conservação de Mamíferos Aquáticos (ICMBio/CMA), FOCUS Diagnóstico Veterinário e ao Instituto Biota de Conservação.

\section{Referências}

Albuquerque-Filho, A.P.L.D. Pneumonia lipóide exógena: importância da história clínica no diagnóstico. Jornal Brasileiro de Pneumologia, 32(6): 596-598, 2006.

Attademo, F.L.N.; Oliveira, R.E.M.; Sousa, G.P.; Luna, F.O. Doenças infecciosas e não infecciosas nos peixes-bois do Brasil. Acta Scientiae Veterinariae, 48(1): 1-7, 2020a.

Attademo, F.L.N.; Luna, F.O.; Sousa, G.P.; Freire, A.C.B.; Balensiefer, D.C.; Oliveira, R.E.M. Piotórax em peixe-boi-marinho (Trichechus manatus) no Brasil. Acta Scientiae Veterinariae, 48(1): 1-7, $2020 \mathrm{~b}$.

Bachman, K.C.; Irvine, A.B. Composition of milk from the Florida manatee, Trichechus manatus latirostris. Comparative Biochemistry and Physiology 62(4): 873878, 1979.

Balensiefer, D.C.; Attademo, F.L.N.; Souza, G.P.; Freire, A.C.B.; Cunha, F.A.G.C.; Alencar, A.E.B.; Silva, F.J.L.; Luna, F.O. Three decades of Antillean manatee (Trichechus manatus manatus) strandings along the Brazilian coast. Tropical Conservation Science, 10(1): 1-9, 2017.

Borges, J.C.G.; Freire, A.C.B.; Attademo, F.L.N; Lima, I.S.; Anzolin, D.G.; Carvalho, P.S.M.; Vergara-Parente, J.E. Growth pattern differences of captive born Antillean manatee (Trichechus manatus) calves and those rescued in the Brazilian Northeastern coast. Journal of Zoo and Wildlife Medicine, 43(3): 494-500, 2012.

BRASIL. Instituto Chico Mendes de Conservação da Biodiversidade. Portaria ${ }^{\circ} 299$ de 4 de abril de 2018. Disponível em: <https://www.icmbio.gov.br/portal/faunabras ileira/planos-de-acao/9327-plano-de-acaonacional-para-a-conservacao-peixe-boimarinho>. Acesso em 15 abr. 2021. 
Buergelt, C.D.; Bonde, R.K.; Beck, C.A.; O'shea, T.J. Pathologic findings in manatees in Florida. Journal of the American Veterinary Medical Association, 185(11): 1331-1334, 1984.

Caswell, J.L.; Williams, K.J. Respiratory system. In: Maxie, M.G. (ed.) Jubb, Kennedy \& Palmer's pathology of domestic animals. Philadelphia: W. B. Saunders, 2007. p.523653.

Freire, A.C.B.; Marmontel, M.; Luna, F.O.; Attademo, F.L.N. Underwater feeding bottle: a new tool in the rehabilitation of manatee calves. Aquatic Mammals, 47(2): 181-184, 2021.

Gage, L.J.; Walsh, M. Hand-rearing and artificial milk formulas. In: Gulland, F.M.D.; Dierauf, L.A.; Whitman, K.L. CRC handbook of marine mammal medicine. New York: CRC Press, 2018. p.739-755.

Gerlach, T.J.; Sadler, V.M.; Ball, R.L. Conservative management of pneumothorax and pneumoperitoneum in two Florida manatees (Trichechus manatus latirostris). Journal of Zoo and Wildlife Medicine, 44(4): 996-1001, 2013.

Lehmhuhl, R.C.; Vulcano, L.C. Tomografia computadorizada. In: Cubas, Z.S.; Silva, J.C.R.; Catão-Dias, J.L. Tratado de animais selvagens: medicina veterinária. São Paulo: Roca, 2014. p.1768-1781.

Luna, F.O.; Passavante, J.Z.O. Projeto peixeboi/ICMBio. 30 Anos de conservação de uma espécie ameaçada. $1^{\text {a }}$ ed. Brasília: ICMBio, 2010. 108p.

Luna, F.O.; Balensiefer, D.C.; Fragoso, A.B.; Stephano, A.; Attademo, F.L.N. Trichechus manatus Linnaeus, 1758. In: Instituto Chico Mendes de Conservação da Biodiversidade. (Org.). Livro Vermelho da Fauna Brasileira Ameaçada de Extinção: Volume II -Mamíferos. Brasília: ICMBio, 2018. p.103-109.

Makara, M.; Shimada, A.; Kawamura, N.; Murase, T.; Morita, T. Aspiration pneumonia as a cause of neonatal death in three captive bottlenose dolphins (Tursiops truncates). Journal of Veterinary Medical Science, 69(3): 325-327, 2007.

Normande, I.C.; Attademo, F.L.N.; Luna, F.O. Antillean manatee release program in Brazil. In: Soorae, P.S. (ed.). Global reintroduction perspectives: 2016. Casestudies from around the globe. Switzerland: IUCN/SSC Reintroduction Specialist Group and Environment Agency-Abu Dhabi, 2016. p.162-165.

Pérez-Accino, J.; Liuti, T.; Pecceu, E.; Cazzini, P. Endogenous lipoid pneumonia associated with pulmonary neoplasia in three dogs. Journal of Small Animal Practice, 62(3): 223-228. 2021.

Rosen, D.A.S.; Worthy, G.A.J. Nutrition and energetics. In: Gulland, F.M.D.; Dierauf, L.A.; Whitman, K.L. CRC handbook of marine mammal medicine. New York: CRC Press, 2018. p.695-738.

Ruoppolo, V.; Vanstreels, R.E.T.; Marigo, J.; Catão-Dias, J.L. Unusual incidence of chronic pneumonia associated with cholesterol deposits in stranded and by caught Franciscanas Pontoporia blainvillei. Diseases of Aquatic Organisms, 92(1): 7581, 2010.

Saunders, J.; Schwarz, T. Principles of CT Image Interpretation. In: Schwarz, T.; Saunders, J. Veterinary Computed Tomography. $1^{\text {st }} \mathrm{ed}$. Nova Jersey: Wiley-Blackwell, 2011. p. 2934.

Tidwel, A.S. Principles of computed tomography and magnetic resonance imaging. In: Thrall, D.E. Textbook of Veterinary Diagnostic Radiology. $5^{\text {rd }}$ ed. St. Louis: Saunders Elsevier, 2007. p.50-77.

Vergara-Parente, J.E.; Sidrim, J.J.C; Pessoa, A.P.B.P.; Parente, C.L.; Marcondes, M.C.C; Teixeira, M.F.; Rocha, M.F.G. Bacterial flora of upper respiratory tract of captive Antillean manatees. Aquatic Mammals, 29(1): 124130, 2003. 